\title{
Work Productivity and Quality-of-Life of Mental Health Patients Attending Neuropsychiatric Hospital, Aro.
}

\author{
Afis A. Agboola ${ }^{1}$, Oluwaseun T. Esan ${ }^{2} \&$ Ajibola T. Soyinka ${ }^{3}$ \\ ${ }^{1}$ Child and Adolescent Mental Health Department, Neuropsychiatric Hospital Aro, Abeokuta, Ogun State, Nigeria \\ ${ }^{2}$ Department of Community Health, Faculty of Clinical Sciences, Obafemi Awolowo University, Ile-Ife, Osun \\ State, Nigeria \\ ${ }^{3}$ Department of Statistics, Federal University of Agriculture, Abeokuta, Ogun State. Nigeria \\ Correspondence: Oluwaseun T. Esan, Department of Community Health, Faculty of Clinical Sciences, Obafemi \\ Awolowo University, Ile-Ife, Nigeria. Tel: 2348037250980. E-mail: seundare2004@gmail.com
}

Received: February 10, 2020 Accepted: March 4, 2020 Online Published: March 19, 2020

doi:10.5539/gjhs.v12n4p81

URL: https://doi.org/10.5539/gjhs.v12n4p81

\begin{abstract}
Introduction: Improving mental health patients' lost work productivity (LWP) may improve their health-related quality of life (HRQOL), and thus reduce their risk for more morbidity and mortality.

Methods: The study investigated the association between the LWP and HRQOL of 284 mental health follow-up patients at a neuro-psychiatric hospital in Nigeria. It was cross-sectional in design with data obtained quantitatively and analysed using the IBM SPSS version 20 at a significance level of $\mathrm{p}<0.05$.

Results: The higher the LWP scores, the worse their level of work productivity but the higher the HRQOL scores, the better their HRQOL. There was a significant relationship between the LWP and HRQOL as every unit improvement in a number of the LWP scales, showed a corresponding significant increase in a number of the patient's HRQOL domains for patients with schizophrenia or bipolar affective disorder. However, patients with depression or mental and behavioural disorders showed no such relationship.
\end{abstract}

Conclusions: The lost work productivity scales and health-related quality of life domains' assessments can be used as monitoring tools by physicians to assess the level of improvement of their patients to treatment. Their roles as prognostic tools can be tested in further studies.

Keywords: Quality-adjusted-life years, health-related quality-of-life, mental health, mental illness, lost work productivity

\section{Introduction}

Health-related Quality-of-Life (HRQoL) refers to a person's perceived physical, mental, emotional and social functioning over a period. It is a description of the feeling of happiness or satisfaction which is beyond the issue of life expectancy. It is important for measuring the impact of chronic diseases, disability, and medical interventions, on the person's well-being. HRQoL can also be used by clinicians and public health experts to generate other measures of disease burden, such as the disability-adjusted life years (DALYs) expressed as the number of years lost due to ill-health, disability or premature death. DALYs is a societal measure of comparing different disease burdens in a population and it differs from other measures like the quality-adjusted life years (QALY) which assesses the quality and quantity of life following a disease condition with or without medical intervention. QALY is an individual and not a societal measure unlike DALYs (Salomon, 2014). However, both measures are useful in prioritising the allocation of resources to different disease conditions and treatment modalities in resource-limited countries.

Mental illnesses are often chronic and are associated with poor quality of life. People with major mental illnesses such as psychosis experience symptoms ranging from hallucinations, thought disturbance, and delusions. These symptoms could interfere directly with day to day living conditions through their impact on behaviour, concentration, memory or decision making (Gee, Pearce, \& Jackson, 2003; Cook \& Chambers, 2009). Mental illnesses can thus impair the productivity and efficiency of a worker, which may in turn negatively affect the overall productive capacity of their organization (Chang, Hong, \& Cho, 2012). 
Five of the ten leading causes of disability worldwide are mental health problems and they constitute major public health problems among high and low-income countries. These problems cut across age, gender and social strata (Murray \& Lopez, 2013). Patients with mental illness also have high disability-adjusted life years compared with other chronic conditions like cardiovascular or respiratory diseases (Gureje, Chisholm, Kola, Lasebikan, \& Saxena, 2007). Specifically, mental illness accounts for about $40 \%$ of morbidity compared with diabetes which accounts for $2 \%$ (Kessler et al., 2005).

Work productivity is a measure of the efficiency of a worker and it could be evaluated in terms of the amount of output produced in a specific period. Work productivity is closely related to the physical, mental, and emotional states of an individual on a particular job with each of the parameters contributing a significant percentage to overall productivity (McTernan, Dollard, \& LaMontagne, 2013). Depending on the age of onset and duration of a mental health disorder, an individual's working capacity may be significantly reduced with a resultant loss in productivity. This loss of work productivity could result from one or a combination of being absent from work due to the illness, loss of concentration because of ill health; poor decision making; deterioration in planning and control of work or total loss of productivity of the affected individual with mental health problem (Noblet \& LaMontagne, 2006).

Studies have shown that huge economic losses are suffered as a result of mental disorders that are complicated by disability or work absenteeism (Greenberg et al, 2003). However, several studies have also reported that improved general health conditions exert a direct impact on economic growth by increasing worker productivity (Woo et al., 2011). Poor mental health status of individuals could create tension and conflicts among colleagues as well as degenerate into poor customer relationship, and these may negatively affect overall productivity in the organization. Environmental factors within the organization such as enhanced occupational safety are essential towards ensuring effective work productivity among workers with unstable mental health conditions (Dewa, Hoch, Nieuwenhuijsen, Parikh, \& Sluiter, 2019) With several millions of people around the world afflicted with severe mental illness, the resultant effects of mental illness on work productivity and quality of life may soon become a public health emergency (Burton, Schultz, Chen, \& Edington, 2008).

Health-related quality of life assessments have been done for patients with different mental illness and other related conditions in Nigeria (Adelufosi, Adebowale, Abayomi, \& Mosanya, 2012), (Adewuya \& Makanjuola, 2010), (Olusina \& Ohaeri, 2003). However, no study had compared work productivity with quality of life among people with different psychiatric diagnoses attending follow up clinics in Nigeria. Knowledge of this may be a measure of treatment success in patients with mental illness and may guide the healthcare providers in treatment decision making in a country with limited resources. This study was aimed at assessing how patients with mental illness attending follow up outpatient clinics at the Neuropsychiatric Hospital, Aro fared in their health-related quality of life and level of work productivity across the different diagnostic categories. Association between their quality of life and level of work productivity was also determined.

\section{Methods}

\subsection{Study Area, Study Design and Study Population}

A descriptive cross-sectional study was conducted among patients aged $\geq 18$ years visiting the outpatient clinic at the Neuropsychiatric Hospital Abeokuta, Nigeria. Inclusion criteria were patients that had been involved in any form of academic or economic activity after discharge from the ward admission in the hospital. Patients with acute medical or mental illness that may impair their full participation during the period of the research were excluded from the study.

\subsection{Sample size and sampling technique}

A sample size of 342 respondents was determined using the Cochran formula for single proportions with a $66.7 \%$ proportion of recently discharged Nigerian psychiatric patients who had an average subjective quality of life after being assessed using the WHOQOL tool (Olusina \& Ohaeri, 2003). The accepted error margin was 5\% at a 95\% confidence limit. Patients were recruited via a systematic random sampling technique among those who met the study criteria.

\subsection{Data Collection and Data Management}

The socio-demographic characteristics of respondents, mental health diagnosis level of work limitation, quality of life (QOL) and other measures were recorded for the respondents after obtaining consent. Respondents' level of work productivity was assessed using the work limitation questionnaire (WLQ) (Lerner et al., 2003). The WLQ is available in the 25 -item version and a modified 8-item short-form version (WLQ-SF). However, the WLQ-SF was used in this study. Responses on WLQ-SF were divided into four work limitations or loss of productivity (LoP) 
subscales. These include the Time Management scale which consists of five items addressing the difficulty in managing time and scheduling demands abbreviated as LoP1. The Physical Demands scale contains six items covering a person's ability to perform job tasks that involve physical strength, stamina, movement, coordination and flexibility abbreviated as LoP2. The Mental/Interpersonal scale has nine items that assess cognitive job tasks and on-the-job social interactions abbreviated as LoP3. The Output Demands scale has five items concerning reduced work quantity and quality abbreviated as LoP4. Scale scores range from 0 (limited none of the time) to 100 (limited all of the time). The score represents the amount of time in the last 2 weeks before the interview that the respondents' were limited in their job or regular work activity. In addition to the scale score, a total WLQ index score can be calculated and converted into an estimate of productivity loss.

The World Health Organization quality of life questionnaire-Bref version (W.H.O QOL-BREF) was used to measure the HRQOL of respondents on follow up treatment for mental illnesses. This is a 26 item, interviewer-administered questionnaire with cross-cultural application, and it assesses the subjective quality of life of patients over the preceding 2 weeks. The HRQOL of life was measured across four domains namely the physical domain (TD1) which assesses patients' mobility status, their performance with daily activities, how well they cope with pain, the quality of their sleep, functional capacity, and energy.

The psychological domain (TD2) assesses patients' self-image and self-esteem, positive attitudes and absence of negative thoughts, ability to learn and ability to concentrate well, presence of a good memory and their mental health status. The social relationship domain (TD3) assesses their inter-personal relationships, the existence of social support and their sex life. While the environmental health domain (TD4) assesses the physical environment where they live, such as exposures to noise and air pollutions, their accessibility to health care and social services, recreation and the safety of their environment. Their ability to acquire new skills and knowledge as well as the financial resources at their disposal for these are all addressed in this domain.

The 4 domains are scored individually, labeled, and transformed to a 0 to 100 scale used to interpret and compare to other validated instruments such as the main WHOQOL-100. A higher score indicates better health.

\subsection{Data Analysis}

Data analysis was done using SPSS version 17 with the level of statistical significance set at $>0.05$. The outcome variable is the patient's quality of life. Expectedly, an inverse relationship should exist between the quality of life and lost work productivity scores. Independent t-tets and ANOVA were used appropriately to compare the patients' lost work productivity and quality of life mean scores. Pearson's Correlation statistics was used to determine the relationship between these two scores and logistic regression was used to quantify how much an increase in quality of life impacted the patient's lost productivity scores. The Internal consistency of the variables assessing the respondents' work productivity and Health-related quality of life had Cronbach's alpha scores of 0.9 and 0.873 respectively. Ethical approval was obtained from the ethical review board committee of the Neuropsychiatric Hospital Aro, Abeokuta, Ogun State.

There are no conflicts of interest in this study. The research was self-funded by the authors and conducted strictly for an academic purpose. All the authors take full responsibility for the study. They were all involved in the design of the study, its implementation, and analysis, the development of the manuscript and approved the final submitted manuscript. The manuscript has not been submitted elsewhere for publication and there are no such intentions.

\section{Results}

A total of 284 out of 342 recruited patients fully participated in the study giving an $83 \%$ response rate. There was a higher proportion of male patients, $210(73.9 \%)$ who were aged 25years and above, 240 (84.5\%). The mean age of the respondents was 33 years \pm 8.78 . More than half of them were either divorced, widowed, separated or yet to be married. More than three-quarters of the respondents, 226 (79.6\%) were gainfully employed with a reported median income of 25,$000 ; 00$. They were in varied occupations with $74(26.1 \%)$ as artisans, $72(25.4 \%)$ as traders and $64(22.4 \%)$ as skilled professionals. (See Table 1$)$ 
Table 1. Socio-demographic characteristics of respondents

\begin{tabular}{|c|c|c|}
\hline Variables & Frequency $(\mathrm{N}=284)$ & Percentage (100\%) \\
\hline \multicolumn{3}{|l|}{ Sex } \\
\hline Female & 74 & 26.1 \\
\hline Male & 210 & 73.9 \\
\hline \multicolumn{3}{|l|}{ Age } \\
\hline 18-24 (young people) & 44 & 15.5 \\
\hline \multicolumn{3}{|l|}{25} \\
\hline 25 and above & 240 & 84.5 \\
\hline Mean age & \multicolumn{2}{|l|}{33.16 years (SD 8.78) } \\
\hline \multicolumn{3}{|l|}{ Religion } \\
\hline Christianity & 189 & 66.5 \\
\hline Islam & 95 & 33.5 \\
\hline \multicolumn{3}{|l|}{ Marital status } \\
\hline Divorced/separated/windowed & 10 & 3.5 \\
\hline Married & 106 & 37.3 \\
\hline Single & 168 & 59.2 \\
\hline \multicolumn{3}{|l|}{ Educational Status } \\
\hline Primary & 11 & 3.9 \\
\hline Junior secondary & 13 & 4.6 \\
\hline Senior secondary & 149 & 52.5 \\
\hline Tertiary/Postgraduate & 111 & 39.1 \\
\hline \multicolumn{3}{|l|}{ Ethnicity } \\
\hline Hausa & 5 & 1.8 \\
\hline Ibo & 56 & 19.7 \\
\hline Yoruba & 219 & 77.1 \\
\hline Others & 4 & 1.4 \\
\hline \multicolumn{3}{|l|}{ Occupation } \\
\hline Artisan/Technician & 74 & 26.1 \\
\hline Government employed & 53 & 18.7 \\
\hline Private employment & 10 & 3.5 \\
\hline Professionals & 64 & 22.5 \\
\hline Students & 6 & 2.1 \\
\hline Sales/Business & 72 & 25.4 \\
\hline Uniformed Officers & 5 & 1.8 \\
\hline \multicolumn{3}{|l|}{ Currently working } \\
\hline Yes & 226 & 79.6 \\
\hline No & 58 & 20.4 \\
\hline \multicolumn{3}{|l|}{ Patients' monthly income } \\
\hline $1000-99000(\$ 2.8-\$ 275)$ & 205 & 72.2 \\
\hline Above $100,000(\geq \$ 277.8)$ & 79 & 27.8 \\
\hline Median income & \multicolumn{2}{|c|}{25000 (USD 69.4) } \\
\hline
\end{tabular}


Schizophrenia was the major disorder 109 (38.4\%) diagnosed among the patients, followed closely by seizure disorder with co-morbid psychosis $81(28.5 \%)$. Mental and behavioural disorders had the least proportion of patients diagnosed with it, $13(4.6 \%)$ as shown in (Figure 1).

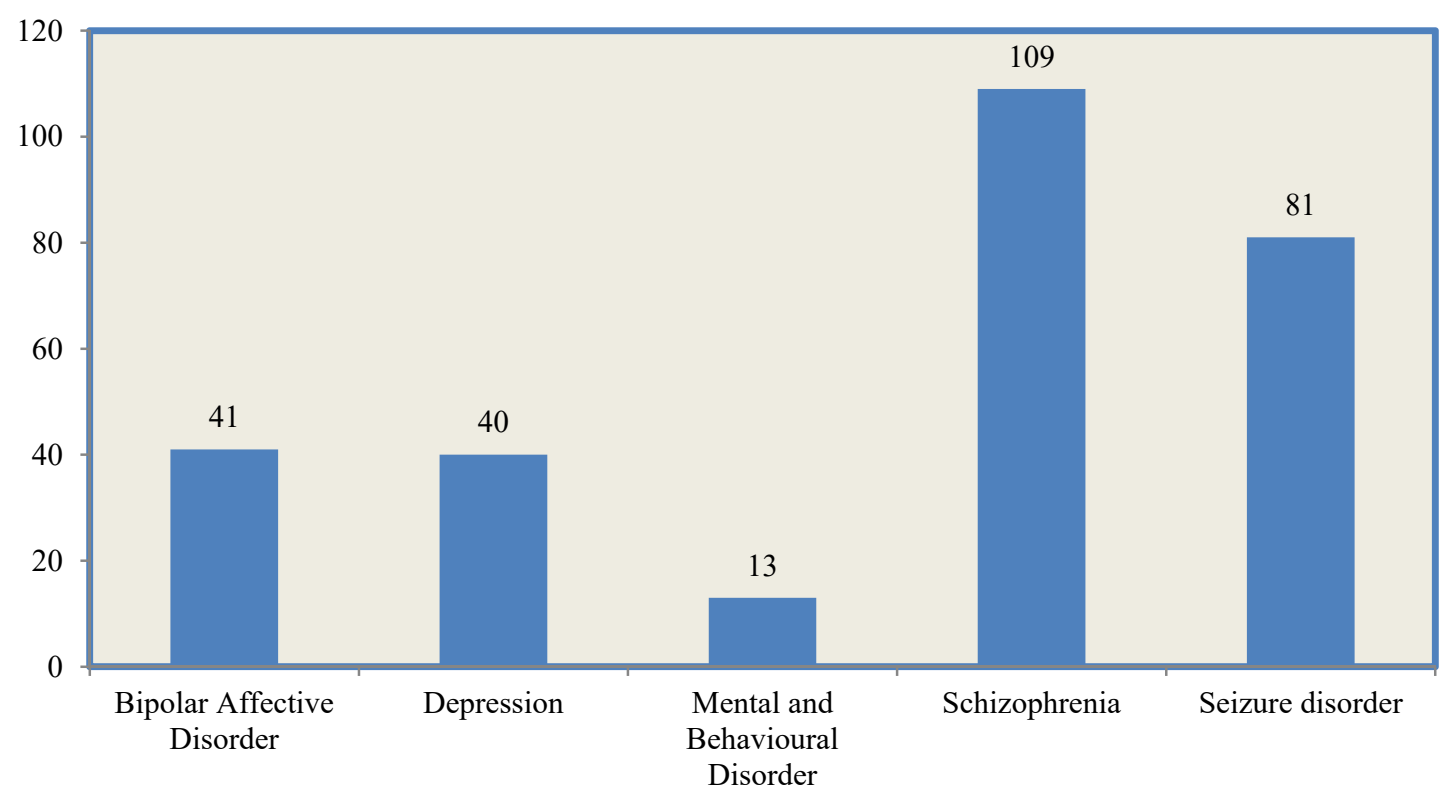

Figure 1. Distribution of ICD 10 Diagnosis of mental illnesses among respondents

The extent of reduced work productivity of respondents depending on if they were currently engaged in productive activities in the last 12 months or not is shown in Table 2. Although all respondents in the two groups reported reduced work productivities despite receiving treatment for mental illness, the group of respondents who were not currently employed reported more significant reduction in time management LoP1, physical demand task LoP2 and overall output demand scale, LoP4. (See Table 2)

Table 2. Comparison of work limitation scores across four domains based on employment status

\begin{tabular}{|c|c|c|c|c|c|c|}
\hline Categories & $\begin{array}{l}\text { Employment } \\
\text { Status }\end{array}$ & $\mathbf{N}$ & Means & $\begin{array}{l}\text { Standard } \\
\text { Deviation }\end{array}$ & \begin{tabular}{llr} 
Test of & \multicolumn{2}{c}{ statistical } \\
significance & degree of \\
freedom (df) & &
\end{tabular} & P-value \\
\hline \multirow{2}{*}{$\begin{array}{l}\text { Time management Scale } \\
\text { (LoP1) }\end{array}$} & $\begin{array}{l}\text { Currently } \\
\text { employed }\end{array}$ & 226 & 9.5183 & 17.7508 & \multirow{2}{*}{ t-test= $-4.21(66,282)$} & \multirow{2}{*}{$\begin{array}{l}\mathrm{p} \\
<0.0001\end{array}$} \\
\hline & $\begin{array}{l}\text { Not currently } \\
\text { employed }\end{array}$ & 58 & 27.8846 & 31.9520 & & \\
\hline \multirow{2}{*}{$\begin{array}{l}\text { Physical Demand Scale } \\
\text { (LoP2) }\end{array}$} & $\begin{array}{l}\text { Currently } \\
\text { employed }\end{array}$ & 226 & 34.82 & 35.848 & \multirow{2}{*}{$\mathrm{t}$-test $=-2.12(282)$} & \multirow{2}{*}{$\mathrm{p}=0.035$} \\
\hline & $\begin{array}{l}\text { Not currently } \\
\text { employed }\end{array}$ & 58 & 45.91 & 34.615 & & \\
\hline \multirow{2}{*}{$\begin{array}{l}\text { Mental Interpersonal } \\
\text { Demand Scale (LoP3) }\end{array}$} & $\begin{array}{l}\text { Currently } \\
\text { employed }\end{array}$ & 226 & 19.4690 & 26.2963 & \multirow{2}{*}{$\mathrm{t}$-test $=-1.83(282)$} & \multirow{2}{*}{$\mathrm{p}=0.069$} \\
\hline & $\begin{array}{l}\text { Not currently } \\
\text { employed }\end{array}$ & 58 & 26.7241 & 29.5663 & & \\
\hline \multirow{2}{*}{$\begin{array}{l}\text { Output } \\
\text { (LoP4) }\end{array}$} & $\begin{array}{l}\text { Currently } \\
\text { employed }\end{array}$ & 226 & 12.1681 & 22.0139 & \multirow{2}{*}{ t-test $=-2.41(76,785)$} & \multirow{2}{*}{$\mathrm{p}=0.018$} \\
\hline & $\begin{array}{l}\text { Not currently } \\
\text { employed }\end{array}$ & 58 & 21.5517 & 27.4854 & & \\
\hline
\end{tabular}

Note. Employment status could mean involvement in a paid job or engagement in academic or productive activities. 
Table 3 showed respondents with varying degrees of work limitation as a result of different psychiatric diagnoses. Comparing different diagnoses, only patients with mental and behavioural disorders due to psychoactive substance use had significant differences in work limitation in their domains, $F=34.241 \mathrm{df}=3,102 \mathrm{p}$-value $=0.001$. Also, respondents diagnosed with depression had the least mean scores for all levels of work productivity. (See Table 3)

Table 3. Comparison of lost work productivity domains across psychiatric diagnoses

\begin{tabular}{|c|c|c|c|c|c|c|}
\hline $\begin{array}{l}\text { Disease } \\
\text { Categories }\end{array}$ & Lost work productivity domains & $\mathbf{N}$ & Means & $\begin{array}{l}\text { Standard } \\
\text { Deviation }\end{array}$ & $\begin{array}{l}\text { Test of Statistical } \\
\text { significance } F(d f)\end{array}$ & P-values \\
\hline \multirow{4}{*}{$\begin{array}{l}\text { Bipolar } \\
\text { Affective } \\
\text { Disorder }\end{array}$} & LP 1-Time management & 41 & 23.684 & 3.285 & \multirow{4}{*}{$2.006(3,116)$} & \multirow{4}{*}{0.117} \\
\hline & LP 2- Physical Demands Scale & 41 & 42.310 & 35.888 & & \\
\hline & $\begin{array}{l}\text { LP 3- Mental Interpersonal Demand } \\
\text { Scale }\end{array}$ & 41 & 23.171 & 2.585 & & \\
\hline & LP 4- Output Demand Scale & 41 & 21.951 & 2.574 & & \\
\hline \multirow{4}{*}{ Depression } & LP 1-Time management & 40 & 6.410 & 11.059 & \multirow{4}{*}{$0.893(3,90)$} & \multirow{4}{*}{0.4480} \\
\hline & LP 2- Physical Demands Scale & 40 & 26.970 & 30.965 & & \\
\hline & $\begin{array}{l}\text { LP 3- Mental Interpersonal Demand } \\
\text { Scale }\end{array}$ & 40 & 15.000 & 25.192 & & \\
\hline & LP 4- Output Demand Scale & 40 & 18.750 & 27.003 & & \\
\hline \multirow{4}{*}{$\begin{array}{l}\text { Mental and } \\
\text { Behavioural } \\
\text { Disorder }\end{array}$} & LP 1-Time management & 13 & 16.667 & 28.867 & \multirow{4}{*}{$34.241(3,102)$} & \multirow{4}{*}{0.0001} \\
\hline & LP 2- Physical Demands Scale & 13 & 45.830 & 35.086 & & \\
\hline & $\begin{array}{l}\text { LP 3- Mental Interpersonal Demand } \\
\text { Scale }\end{array}$ & 13 & 23.077 & 25.944 & & \\
\hline & LP 4- Output Demand Scale & 13 & 23.077 & 16.013 & & \\
\hline \multirow{4}{*}{ Schizophrenia } & LP 1-Time management & 109 & 11.905 & 18.376 & \multirow{4}{*}{$0.210(3,108)$} & \multirow{4}{*}{0.8892} \\
\hline & LP 2- Physical Demands Scale & 109 & 34.810 & 36.303 & & \\
\hline & $\begin{array}{l}\text { LP 3- Mental Interpersonal Demand } \\
\text { Scale }\end{array}$ & 109 & 19.954 & 26.304 & & \\
\hline & LP 4- Output Demand Scale & 109 & 24.541 & 30.615 & & \\
\hline \multirow{4}{*}{$\begin{array}{l}\text { Seizure } \\
\text { Disorder }\end{array}$} & LP 1-Time management & 81 & 12.171 & 22.911 & \multirow{3}{*}{$0.667(3,117)$} & \multirow{4}{*}{0.5739} \\
\hline & LP 2- Physical Demands Scale & 81 & 41.030 & 36.922 & & \\
\hline & $\begin{array}{l}\text { LP 3- Mental Interpersonal Demand } \\
\text { Scale }\end{array}$ & 81 & 23.765 & 29.818 & & \\
\hline & LP 4- Output Demand Scale & 81 & 27.778 & 31.628 & & \\
\hline
\end{tabular}

The mean quality of life scores across each domain for a corresponding psychiatric diagnosis is shown in Table 4 . The higher the score on each domain, the better the quality of life of the respondents who had received treatment for mental illness. Mental and behavioral disorders due to psychoactive substance use had the highest scores across all the QOL domains while patients with the diagnosis of schizophrenia had the lowest scores in each of the domains. There were statistically significant differences in the mean quality of life scored by patients being managed for the listed mental health conditions in the TD1, assessing their physical health, $(\mathrm{p}=0.004)$; the TD2 assessing their psychological health, $(\mathrm{p}=0.031)$ and the TD4 assessing their health within their immediate environment, $(\mathrm{p}=0.01)$. (See Table 4) 
Table 4. Quality of life across each domain and selected mental health disorders

\begin{tabular}{|c|c|c|c|c|c|c|}
\hline QOL Domains & Disease Categories & $\mathbf{N}$ & Means & $\begin{array}{l}\text { Standard } \\
\text { Deviation }\end{array}$ & $\begin{array}{l}\text { Test of Statistical } \\
\text { significance } F(\mathbf{d f})\end{array}$ & P-values \\
\hline \multirow{5}{*}{$\begin{array}{l}\text { TDI- Physical } \\
\text { Domain }\end{array}$} & Bipolar Affective Disorder & 41 & 63.3275 & 11.792 & \multirow{5}{*}{$3.889(4,279)$} & \multirow{5}{*}{0.004} \\
\hline & Depression & 40 & 61.8750 & 9.617 & & \\
\hline & Mental Behavioural Disorder & 13 & 68.6813 & 14.808 & & \\
\hline & Schizophrenia & 109 & 59.9934 & 15.664 & & \\
\hline & Acute Psychosis Disorder & 81 & 66.7108 & 9.924 & & \\
\hline \multirow{5}{*}{$\begin{array}{l}\text { TD2- } \\
\text { Psychological } \\
\text { Domain }\end{array}$} & Bipolar Affective Disorder & 41 & 57.5203 & 14.767 & \multirow{5}{*}{$2.702(4,279)$} & \multirow{5}{*}{0.031} \\
\hline & Depression & 40 & 61.25 & 10.083 & & \\
\hline & Mental Behavioural Disorder & 13 & 65.7051 & 10.644 & & \\
\hline & Schizophrenia & 109 & 57.7982 & 16.009 & & \\
\hline & Acute Psychosis Disorder & 81 & 63.5802 & 14.699 & & \\
\hline \multirow{5}{*}{$\begin{array}{l}\text { TD3- Social } \\
\text { relationship } \\
\text { domain }\end{array}$} & Bipolar Affective Disorder & 41 & 65.2033 & 18.827 & \multirow{5}{*}{$0.907(4,279)$} & \multirow{5}{*}{0.461} \\
\hline & Depression & 40 & 68.5 & 11.195 & & \\
\hline & Mental Behavioural Disorder & 13 & 73.33 & 15.154 & & \\
\hline & Schizophrenia & 109 & 65.9327 & 18.4 & & \\
\hline & Acute Psychosis Disorder & 81 & 69.1358 & 20.178 & & \\
\hline \multirow{5}{*}{$\begin{array}{l}\text { TD4- } \\
\text { Environmental } \\
\text { Domain }\end{array}$} & Bipolar Affective Disorder & 41 & 66.1585 & 14.406 & \multirow{5}{*}{$3.406(4,279)$} & \multirow{5}{*}{0.01} \\
\hline & Depression & 40 & 71.25 & 8.006 & & \\
\hline & Mental Behavioural Disorder & 13 & 74.0385 & 14.561 & & \\
\hline & Schizophrenia & 109 & 66.8807 & 14.542 & & \\
\hline & Acute Psychosis Disorder & 81 & 72.9012 & 13.989 & & \\
\hline
\end{tabular}

TD1: Transformed quality of life score Physical domain 1; TD2: Transformed quality of life score Psychological domain 2; TD3: Transformed quality of life score Social relationship domain 3; TD4: Transformed quality of life score Environment domain 4.

Patients with bipolar affective disorder and schizophrenia showed some significantly low to mild inverse or negative relationship which is as expected between a number of their lost work productivity scales and their quality of life domains. Patients with seizure disorder showed the same relationship only with their lost work productivity time management scale and their quality of life physical and social relationship domains. However, there was a direct or positive, though mild but significant relationship between their quality of life physical domain and their work productivity physical demand scale. Patients with depression showed no significant relationship across all their quality of life domains and lost work productivity scales. While patients with mental and behavioral disorder showed a significant and severe direct or positive relationship between their work productivity physical demand scale and all the domains across their quality of life. Their Mental Interpersonal demand scale also had a similar relationship with their quality of life psychological domain. (See Table 5) 
Table 5: Relationship between work limitation and quality of life domains of respondents across different mental health disorders.

\begin{tabular}{|c|c|c|c|c|c|}
\hline $\begin{array}{l}\text { Disease } \\
\text { Categories }\end{array}$ & Variables & $\begin{array}{l}\text { TD1- } \\
\text { Physical } \\
\text { Domain } \\
\text { rho (p-value) }\end{array}$ & $\begin{array}{l}\text { TD2- } \\
\text { Psychological } \\
\text { Domain } \\
\text { rho (p-value) }\end{array}$ & $\begin{array}{l}\text { TD3- Social } \\
\text { Relationship } \\
\text { Domain } \\
\text { rho (p-value) }\end{array}$ & $\begin{array}{l}\text { TD4- Environmental } \\
\text { Domain } \\
\text { rho (p-value) }\end{array}$ \\
\hline \multirow{4}{*}{$\begin{array}{l}\text { Bipolar } \\
\text { Affective } \\
\text { Disorder }\end{array}$} & $\begin{array}{l}\text { LP 1-Time } \\
\text { management }\end{array}$ & $-.273(0.097)$ & $-.502(.001)^{*}$ & $-.204(219)$ & $-.353(0.030)^{*}$ \\
\hline & $\begin{array}{l}\text { LP 2- Physical } \\
\text { Demands Scale }\end{array}$ & $.039(0.812)$ & $-.144(0.382)$ & $.040(0.810)$ & $-.139(0.398)$ \\
\hline & $\begin{array}{l}\text { LP 3- Mental } \\
\text { Interpersonal }\end{array}$ & $-.240(0.130)$ & $-.509(0.001)$ & $-.314(0.046)$ & $-.472(0.002)^{* *}$ \\
\hline & $\begin{array}{l}\text { LP 4- Output } \\
\text { Demand Scale }\end{array}$ & $-.091(.572)$ & $-.171(.285)$ & $-.078(0.627)$ & $-.289(.066)$ \\
\hline \multirow{4}{*}{ Depression } & $\begin{array}{l}\text { LP } \quad 1 \text {-Time } \\
\text { management }\end{array}$ & $-.307(.057)$ & $-.077(.640)$ & $-.248(.126)$ & $.030(.855)$ \\
\hline & $\begin{array}{l}\text { LP 2- Physical } \\
\text { Demands Scale }\end{array}$ & $.165(.323)$ & $.306(.062)$ & $.284(.134)$ & $.194(.244)$ \\
\hline & $\begin{array}{l}\text { LP 3- Mental } \\
\text { Interpersonal }\end{array}$ & $210(.194)$ & $.212(.188)$ & $-.055(.738)$ & $.310(.052)$ \\
\hline & $\begin{array}{l}\text { LP 4- Output } \\
\text { Demand Scale }\end{array}$ & $.284(.075)$ & $.216(.181)$ & $-.004(.983)$ & $.208(.199)$ \\
\hline \multirow{4}{*}{$\begin{array}{l}\text { Mental and } \\
\text { Behavioural } \\
\text { Disorder }\end{array}$} & $\begin{array}{l}\text { LP } 1 \text {-Time } \\
\text { management }\end{array}$ & $.520(.083)$ & $.502(.096)$ & $.527(.078)$ & $.388(.212)$ \\
\hline & $\begin{array}{l}\text { LP 2- Physical } \\
\text { Demands Scale }\end{array}$ & $.657(.020)^{*}$ & $.716(.009)^{* *}$ & $.613(.034)^{*}$ & $.712(.009)^{* *}$ \\
\hline & $\begin{array}{l}\text { LP 3- Mental } \\
\text { Interpersonal }\end{array}$ & $.644(.018)$ & $.559(.047)^{*}$ & $.495(.086)$ & $.436(.137)$ \\
\hline & $\begin{array}{l}\text { LP 4- Output } \\
\text { Demand Scale }\end{array}$ & $-.181(.554)$ & $-.114(.047)^{*}$ & $-.401(.175)$ & $-.321(.284)$ \\
\hline \multirow{4}{*}{ Schizophrenia } & $\begin{array}{l}\text { LP } \quad 1 \text {-Time } \\
\text { management }\end{array}$ & $-211(.031)$ & $-.146(.138)$ & $-.175(.074)$ & $-.261(.007)^{*}$ \\
\hline & $\begin{array}{l}\text { LP 2- Physical } \\
\text { Demands Scale }\end{array}$ & $.055(.572)$ & $.060(.537)$ & $.198(.041)$ & $.007(.942)$ \\
\hline & $\begin{array}{l}\text { LP 3- Mental } \\
\text { Interpersonal }\end{array}$ & $-.159(.098)$ & $-.043(.656)$ & $-.158(.102)$ & $-.271(.004)^{*}$ \\
\hline & $\begin{array}{l}\text { LP 4- Output } \\
\text { Demand Scale }\end{array}$ & $-.078(.419)$ & $-.030(.757)$ & $.008(.937)$ & $-.115(.234)$ \\
\hline \multirow{4}{*}{$\begin{array}{l}\text { Seizure } \\
\text { Disorder }\end{array}$} & $\begin{array}{l}\text { LP 1-Time } \\
\text { management }\end{array}$ & $-.342(.003)^{*}$ & $-.078(.505)$ & $-.302(.008)^{*}$ & $-.097(.404)$ \\
\hline & $\begin{array}{l}\text { LP 2- Physical } \\
\text { Demands Scale }\end{array}$ & $.258(.023)^{*}$ & $.211(.064)$ & $.207(.069)$ & $.193(.091)$ \\
\hline & $\begin{array}{l}\text { LP 3- Mental } \\
\text { Interpersonal }\end{array}$ & $.089(.427)$ & $.175(.117)$ & $.054(.635)$ & $.190(.089)$ \\
\hline & $\begin{array}{l}\text { LP 4- Output } \\
\text { Demand Scale }\end{array}$ & $.148(.218)$ & $.190(0.090)$ & $.015(.893)$ & $.160(.154)$ \\
\hline
\end{tabular}

* Statistically significant findings.

For patients with bipolar affective disorder, a one-unit increase in their ability to cope well with time management will lead to a $70 \%$ increase in their quality of life psychological health domain. Also, a unit increase in the ability to improve the quantity and quality of their work output is likely to increase their psychological health and environmental health in their quality of life domains by $61 \%$ and $100 \%$ respectively. However, it is less likely to increase their physical health by $65 \%$. All these findings are statistically significant. 
In patients with depression with a unit gain in coping well on their lost work productivity time management scale, will likely significantly increase their quality of life social and relationships domain and environmental health domain by $36 \%$ and $55 \%$. Also, a unit increase in the quantity and quality of work done as measured by their output demand scale will yield a $136 \%$ increase in their environmental health domain such as having more financial resources and better access to health care services.

Patients with mental and behavioural disorder had no significant predictive effects of changes in their lost work productivity scale on any of their quality of life domains. For patients with schizophrenia, a unit increase in their ability to cope better with each of their lost work productivity scales had a significant percent increase only in their quality of life environmental health domain. While for patients with a seizure disorder, a unit increase in their ability to cope well with each of their lost work productivity scales had a significant increase only in their quality of life physical domain. (See Table 6)

Table 6. Predictive effects of work productivity on patients' quality of life

\begin{tabular}{|c|c|c|c|c|c|}
\hline $\begin{array}{ll}\text { Lost } & \text { work } \\
\text { productivity } & \\
\text { by mental health } \\
\text { diagnosis }\end{array}$ & $\begin{array}{l}\text { TD1-Physical } \\
\text { Domain } \\
\text { Coeff. (p-value) }\end{array}$ & $\begin{array}{l}\text { TD2-Psycholofical } \\
\text { Domain } \\
\text { Coeff. (p-value) }\end{array}$ & $\begin{array}{l}\text { TD3 Social } \\
\text { relationship Domain } \\
\text { Coeff. (p-value) }\end{array}$ & $\begin{array}{lr}\text { TD4 } & \text { Environmental } \\
\text { Domain } & \text { Coeff. } \\
\text { (p-value) } & \end{array}$ & $\mathbf{R}^{2}$ \\
\hline \multicolumn{6}{|c|}{ Bipolar Affective Disorder } \\
\hline $\begin{array}{l}\text { LP1-Time } \\
\text { management }\end{array}$ & $-0.001(0.881)$ & $0.696(0.001)^{*}$ & $-0.104(0.645)$ & $0.426(0.199)$ & 0.929 \\
\hline $\begin{array}{l}\text { LP2-Physical } \\
\text { Demands Scale }\end{array}$ & $-0.128(0.803)$ & $0.493(0.266)$ & $-0.296(0.383)$ & $0.845(0.094)$ & 0.839 \\
\hline $\begin{array}{l}\text { LP3-Mental } \\
\text { Interpersonal } \\
\text { Demand Scale }\end{array}$ & $0.284(0.53)$ & $0.142(0.61)$ & $-0.098(0.741)$ & $0.581(0.184)$ & 0.877 \\
\hline $\begin{array}{ll}\text { LP4-Output Demand } \\
\text { Scale }\end{array}$ & $-0.646(0.043)^{*}$ & $0.615(0.01)^{*}$ & $-0.002(0.994)$ & $1.003(0.002)^{*}$ & 0.944 \\
\hline \multicolumn{6}{|l|}{ Depression } \\
\hline $\begin{array}{l}\text { LP1-Time } \\
\text { management }\end{array}$ & $0.263(0.215)$ & $-0.180(0.358)$ & $0.362(0.021)^{*}$ & $0.551(0.005)^{*}$ & 0.985 \\
\hline $\begin{array}{l}\text { LP2-Physical } \\
\text { Demands Scale }\end{array}$ & $0.563(0.367)$ & $-0.698(0.185)$ & $0.074(0.817)$ & $1.003(0.052)$ & 0.897 \\
\hline $\begin{array}{ll}\text { LP3- } & \text { Mental } \\
\text { Interpersonal } & \\
\text { Demand Scale } & \end{array}$ & $0.424(0.496)$ & $-0.201(0.704)$ & $0.561(0.213)$ & $0.167(0.742)$ & 0.9 \\
\hline $\begin{array}{l}\text { LP4-Output Demand } \\
\text { Scale }\end{array}$ & $-0.940(0.195)$ & $-0.091(0.875)$ & $0.607(0.21)$ & $1.364(0.019)^{*}$ & 0.894 \\
\hline \multicolumn{6}{|c|}{ Mental and Behiovral disorder } \\
\hline $\begin{array}{ll}\text { LP1- } & \text { Time } \\
\text { management } & \end{array}$ & $-1.323(0.379)$ & $1.469(0.316)$ & $-0.451(0.741)$ & $1.238(0.378)$ & 0.898 \\
\hline $\begin{array}{l}\text { LP2- Physical } \\
\text { Demands Scale }\end{array}$ & $-1.019(0.655)$ & $1.686(0.450)$ & $0.019(0.993)$ & $0.172(0.935)$ & 0.757 \\
\hline $\begin{array}{lr}\text { LP3- } & \text { Mental } \\
\text { Interpersonal } & \text { Demand } \\
\text { Scale } & \end{array}$ & $-2.303(0.119)$ & $1.316(0.333)$ & $0.209(0.869)$ & $1.709(0.203)$ & 0.912 \\
\hline $\begin{array}{l}\text { LP4- Output Demand } \\
\text { Scale }\end{array}$ & $-2.285(0.067)$ & $0.9(0.414)$ & $0.641(0.54)$ & $1.694(0.131)$ & 0.941 \\
\hline \multicolumn{6}{|l|}{ Schizophrenia } \\
\hline $\begin{array}{l}\text { LP1- } \\
\text { management }\end{array}$ & $0.049(0.769)$ & $-0.026(0.852)$ & $0.177(0.108)$ & $0.778(0.0005)^{*}$ & 0.949 \\
\hline
\end{tabular}




\begin{tabular}{|c|c|c|c|c|c|}
\hline $\begin{array}{l}\text { LP2- Physical } \\
\text { Demands Scale }\end{array}$ & $0.113(0.722)$ & $-0.058(0.824)$ & $-0.209(0.314)$ & $1.056(0.0005)^{*}$ & 0.949 \\
\hline $\begin{array}{lr}\text { LP3- } & \text { Mental } \\
\text { Interpersonal } & \text { Demand } \\
\text { Scale } & \end{array}$ & $0.044(0.86)$ & $-0.058(0.775)$ & $0.102(0.517)$ & $0.86(0.0005)^{*}$ & 0.895 \\
\hline $\begin{array}{ll}\text { LP4-Output Demand } \\
\text { Scale }\end{array}$ & $-0.104(0.678)$ & $-0.042(0.838)$ & $0.12(0.449)$ & $0.972(0.0005)^{*}$ & 0.894 \\
\hline \multicolumn{6}{|l|}{ Seizure disorder } \\
\hline $\begin{array}{ll}\text { LP1- } & \text { Time } \\
\text { management } & \end{array}$ & $1.064(0.005)^{*}$ & $-0.283(0.076)$ & $0.183(0.106)$ & $0.018(0.919)$ & 0.962 \\
\hline $\begin{array}{l}\text { LP2- Physical } \\
\text { Demands Scale }\end{array}$ & $0.881(0.016)^{*}$ & $-0.264(0.431)$ & $-0.277(0.284)$ & $0.547(0.189)$ & 0.802 \\
\hline $\begin{array}{l}\text { LP3- } \\
\text { Interpersonal } \\
\text { Demand Scale }\end{array}$ & $1.120(0.0005)^{*}$ & $-0.218(0.428)$ & $0.261(0.222)$ & $-0.229(0.514)$ & 0.875 \\
\hline $\begin{array}{l}\text { LP4- Output Demand } \\
\text { Scale }\end{array}$ & $0.931(0.001)^{*}$ & $-0.317(0.209)$ & $0.150(0.439)$ & $0.182(0.569)$ & 0.895 \\
\hline
\end{tabular}

\section{Discussion}

Mental illness conditions are leading causes of workplace absenteeism or loss of job opportunities. Major psychiatric disorders like schizophrenia are commonly associated with impairment in social and occupational functioning and in some cases loss of independent life (Whiteford et al., 2013). The majority of respondents in this study were currently employed at the time of the research even though the diagnosis of schizophrenia predominates among them. The study provided us opportunity to assess the effect of their work productivity following response to treatment for their various psychiatric disorders and the resultant effect of these on their health-related quality of life.

The study showed that patients reported varying degrees of working limitation across different psychiatric diagnoses, thus resulting in lost productivity. Respondents who were currently employed had lower work limitation scores compared with those that were unemployed, hence the former fared better. The significant lost productivity of unemployed respondents occurred in three of the four domains; except in the mental interpersonal demand scale when compared with those respondents who were employed. The mental interpersonal demand scale of the two groups (employed and unemployed) showed no statistically significant differences possibly because the two groups both suffered from mental illness, however, higher scores in those unemployed might be as a result of more severe illness among them.

Patients with affective disorder had the highest work limitation scores probably because of impaired concentration and racing thoughts that normally characterize people with this illness. They may be able to complete a given task at a record time but with less precision because of the high energy seen in them (Cook and Chambers 2009). However, the depressive respondents had the lowest work limitation scales because, with treatment, there may be the return of a pleasurable feeling in ones' usual activity like working which may result in better productivity for depressive patients. Psychiatric patients may suffer symptoms exacerbation at a repeated intervals (Roy \& Schurer, 2013), and during this episode, they have difficulties in concentration, lost of insight to reality, lack motivation, impaired in mastering daily routines thus impaired level of productivity (Stewart, Ricci, Chee, Hahn, \& Morganstein, 2003)

Some countries in Europe where people with major psychiatric disorders like schizophrenia are given supervised employment, lost productivity had reportedly made up $64 \%$ of the total costs spent on the care of people with mental illness (SobockJonsson et al 2006). In other studies, the costs due to lost productivity are 2 to 3 times larger than the costs due to health care utilization (Cuijpers et al., 2007; Greenberg et al., 2003; Hawthorne, Cheok, Goldney, \& Fisher, 2003; Sobocki, Jönsson, Angst, \& Rehnberg, 2006). Their lost productivity could result from absenteeism or physical presence at work with lower productivity because of ill health or in some cases total 
withdrawals from the labor market owing to the chronicity of the illness or premature death. Prolonged absence from paid work due to illness can be expected to increase the need to pay for health services and goods out of other household resources (family savings). While the inability to carry out unpaid but productive household activities because of ill-health (including child care, meal preparation etc.) may lead to time being taken off from paid work by another household member. The mechanisms through which it influences current and future consumption are manifold.

Health-related Quality of Life (HRQoL) refers to a person's perceived physical and mental health at a point in time. It is important to measure the impact of both chronic diseases and medical interventions on the patient's well-being to evaluate the effect of the intervention. The result of this study confirmed that mental illness affected the quality of life of the individuals over the course of their illness. Schizophrenic patients in this study reported the lowest quality of life. The social relationship which is part of the quality of life domains assessed is an important area that is usually affected in any major mental illness. for any diagnosis of mental disorder to be made, assessment of social interactions or social relationships is a key criterion to be considered. The subjective experience of mental illness was described as a debilitating burden (Cuijpers et al 2007) Pre-occupation with the symptoms of mental health problems often interferes greatly with the most basic tasks of everyday living (Corring 2005). People diagnosed with psychosis or mania reported that hallucinations and delusions particularly hearing of voices, thought disturbances and paranoia were most debilitating for them with resultant poor quality of life (Gee, L., Pearce, E., \& Jackson, M. 2003). These symptoms could interfere directly with day to day living by affecting their behaviour control, concentration, memory and decision making (Cook \& Chambers, 2009; Gee et al., 2003).

There has been a shift in mental health service delivery with more emphasis on social, occupational and psycho-pharmacological rehabilitation. As a result of these, quality of life and work productivity assessments are essential parameters for observation among mentally ill individuals. This study reported a strong correlation between the quality of life and work limitation with loss of productivity across patients with different psychiatric diagnoses. Lost work productivity may lead to poor quality of life and vice versa. Absence from work due to illness increases financial demands on the household and if the individual suffered a cognitive and social decline from this, it may result in another family member taking time out of their paid job to care for the patient. This is a common scenario in low-income countries like Nigeria where treatment for mental illness is largely an out of pocket expenditure with attendant effects on family savings.

Furthermore, the predictive effects of changes in lost work productivity were measured on patients' health-related quality of life. From this study, the environmental and physical health domains of the health-related quality of life were more reactive to changes in their lost work productivity especially for patients with schizophrenia and seizure disorder. While changes in patients with mental and behavioural disorder had no significant effect on the patient's quality of life. The reason for this may be because this is more of a behavioural disorder with minimal effect on the patient's mental status if the patients were in the abstinence period after treatment of any associated psychosis in the hospital.

Conclusively, quality of life measurement and lost work productivity assessment play an important role in quantifying the patient's morbidity with psychiatric disorders, and in guiding patient management decisions. Emphasis should not be on just keeping patients alive but also enriching their quality of life. There was a strong relationship between loss work productivity and health-related quality of life from this study: For every unit improvement in some of the lost work productivity scale, there was a corresponding significant increase in a number of the patient's quality of life domains. Improvement in the quality of life of a mentally ill individual will also translate to more improvement in their level of work productivity scale in a continuum. Thus, the lost work productivity scales and health-related quality of life domains' assessments can be used as monitoring tools by physicians to assess the level of improvement of their patients to treatment. It can as well serve as prognostic tools. The more the reduction in the lost work productivity scales achieved, the more it is expected to positively impact their health-related quality of life and reduce mortality amongst our patients. It is recommended that similar studies are conducted in other centers among similar patients in Nigeria and other countries. This will confer external validity on the findings of this research.

Limitations observed in this study include the unavailability of baseline data of patients on lost work productivity and quality of life before treatment, as patients were not followed up over time, being a descriptive cross-sectional study. Also, the lack of more objective assessments of patients' function at work and recall bias due to memory distortion may have limited the study findings.

\section{Acknowledgements}

There are no acknolwedgements. 


\section{Competing Interests Statement}

The authors declare that there are no competing or potential conflicts of interest.

\section{References}

Adelufosi, A. O., Adebowale, T. O., Abayomi, O., \& Mosanya, J. T. (2012). Medication adherence and quality of life among Nigerian outpatients with schizophrenia. General Hospital Psychiatry, 34(1), $72-79$. https://doi.org/10.1016/j.genhosppsych.2011.09.001

Adewuya, A. O., \& Makanjuola, R. O. (2010). Subjective life satisfaction and objective living conditions of patients with schizophrenia in Nigeria. Psychiatric Services, 61(3), 314-316. https://doi.org/10.1176/ps.2010.61.3.314

Burton, W. N., Schultz, A. B., Chen, C. Y., \& Edington, D. W. (2008). The association of worker productivity and mental health: A review of the literature. International Journal of Workplace Health Management. https://doi.org/10.1108/17538350810893883

Chang, S. M., Hong, J.-P., \& Cho, M. J. (2012). Economic burden of depression in South Korea. Social Psychiatry and Psychiatric Epidemiology, 47(5), 683-689. https://doi.org/10.1007/s00127-011-0382-8

Cook, S., \& Chambers, E. (2009). What helps and hinders people with psychotic conditions doing what they want in their daily lives. British Journal of Occupational Therapy, 72(6), 238-248. https://doi.org/10.1177/030802260907200602

Cuijpers, P., Smit, F., Oostenbrink, J., de Graaf, R., ten Have, M., \& Beekman, A. (2007). Economic costs of minor depression: a population-based study. Acta Psychiatrica Scandinavica, 115(3), 229-236. https://doi.org/10.1111/j.1600-0447.2006.00851.x

Dewa, C. S., Hoch, J. S., Nieuwenhuijsen, K., Parikh, S. V., \& Sluiter, J. K. (2019). Toward Effective Work Accommodations for Depression: Examining the Relationship Between Different Combinations of Depression Symptoms and Work Productivity Losses. Journal of Occupational and Environmental Medicine. https://doi.org/10.1097/JOM.0000000000001486

Gee, L., Pearce, E., \& Jackson, M. (2003). Quality of life in schizophrenia: a grounded theory approach. Health and Quality of Life Outcomes, 1(1), 31. https://doi.org/10.1186/1477-7525-1-31

Greenberg, P. E., Kessler, R. C., Birnbaum, H. G., Leong, S. A., Lowe, S. W., Berglund, P. A., \& Corey-Lisle, P. K. (2003). The economic burden of depression in the United States: how did it change between 1990 and 2000 ? The Journal of Clinical Psychiatry, 64(12), 1465-1475.

Gureje, O., Chisholm, D., Kola, L., Lasebikan, V., \& Saxena, S. (2007). Cost-effectiveness of an essential mental health intervention package in Nigeria. World Psychiatry, 6(1), 42-48.

Hawthorne, G., Cheok, F., Goldney, R., \& Fisher, L. (2003). The excess cost of depression in South Australia: a population-based study. The Australian and New Zeaanld Journal of Psychiatry, 37(3), 362-373. https://doi.org/10.1046/j.1440-1614.2003.01189.x

Kessler, R. C., Berglund, P., Demler, O., Jin, R., Merikangas, K. R., \& Walters, E. E. (2005, June). Lifetime prevalence and age-of-onset distributions of DSM-IV disorders in the national comorbidity survey replication. Archives of General Psychiatry, 62, 593-602. https://doi.org/10.1001/archpsyc.62.6.593

Lerner, D., Amick, B. C., Lee, J. C., Rooney, T., Rogers, W. H., Chang, H., \& Berndt, E. R. (2003). Relationship of employee-reported work limitations to work productivity. Medical Care, 41(5), 649-659. https://doi.org/10.1097/01.MLR.0000062551.76504.A9

McTernan, W. P., Dollard, M. F., \& LaMontagne, A. D. (2013). Depression in the workplace: An economic cost analysis of depression-related productivity loss attributable to job strain and bullying. Work and Stress, 27(4), 321-338. https://doi.org/10.1080/02678373.2013.846948

Murray, C. J. L., \& Lopez, A. D. (2013). Measuring the global burden of disease. New England Journal of Medicine, 369, 448-457. https://doi.org/10.1056/NEJMra1201534

Noblet, A., \& LaMontagne, A. D. (2006). The role of workplace health promotion in addressing job stress. Health Promotion International. https://doi.org/10.1093/heapro/dal029

Olusina, A. K., \& Ohaeri, J. U. (2003). Subjective quality of life of recently discharged Nigerian psychiatric patients. Social Psychiatry and Psychiatric Epidemiology, 38(12), 707-714. https://doi.org/10.1007/s00127-003-0691-7 
Roy, J., \& Schurer, S. (2013). Getting stuck in the blues: persistence of mental health problems in Australia. Health Economics, 22(9), 1139-1157. https://doi.org/10.1002/hec.2967

Salomon, J. A. (2014). Disability-Adjusted Life Years. In Encyclopedia of Health Economics (pp. 200-203). https://doi.org/10.1016/B978-0-12-375678-7.00511-3

Sobocki, P., Jönsson, B., Angst, J., \& Rehnberg, C. (2006). Cost of depression in Europe. The Journal of Mental Health Policy and Economics, 9(2), 87-98.

Stewart, W. F., Ricci, J. A., Chee, E., Hahn, S. R., \& Morganstein, D. (2003). Cost of lost productive work time among US workers with depression. JAMA, 289(23), 3135-3144. https://doi.org/10.1001/jama.289.23.3135

Whiteford, H. A., Degenhardt, L., Rehm, J., Baxter, A. J., Ferrari, A. J., Erskine, H. E., ... Vos, T. (2013). Global burden of disease attributable to mental and substance use disorders: Findings from the Global Burden of Disease Study 2010. The Lancet, 382(9904), 1575-1586. https://doi.org/10.1016/S0140-6736(13)61611-6

Woo, J. M., Kim, W., Hwang, T. Y., Frick, K. D., Choi, B. H., Seo, Y. J., ... Park, Y. L. (2011). Impact of depression on work productivity and its improvement after outpatient treatment with antidepressants. Value in Health, 14(4), 475-482. https://doi.org/10.1016/j.jval.2010.11.006

\section{Copyrights}

Copyright for this article is retained by the author(s), with first publication rights granted to the journal.

This is an open-access article distributed under the terms and conditions of the Creative Commons Attribution license (http://creativecommons.org/licenses/by/4.0/). 